\title{
Charge transport in graphene with resonant scatterers
}

\author{
M. Titov, ${ }^{1,2}$ P. M. Ostrovsky, ${ }^{3,4}$ I. V. Gornyi, ${ }^{3,5,2}$ A. Schuessler, ${ }^{3}$ and A. D. Mirlin ${ }^{3,6,7,2}$ \\ ${ }^{1}$ School of Engineering \& Physical Sciences, Heriot-Watt University, Edinburgh EH14 4AS, UK \\ ${ }^{2}$ DFG Center for Functional Nanostructures, Karlsruhe Institute of Technology, 76128 Karlsruhe, Germany \\ ${ }^{3}$ Institut für Nanotechnologie, Karlsruhe Institute of Technology, 76021 Karlsruhe, Germany \\ ${ }^{4}$ L. D. Landau Institute for Theoretical Physics RAS, 119334 Moscow, Russia \\ ${ }^{5}$ A. F. Ioffe Physico-Technical Institute, 194021 St. Petersburg, Russia. \\ ${ }^{6}$ Institut für Theorie der kondensierten Materie, Karlsruhe Institute of Technology, 76128 Karlsruhe, Germany \\ ${ }^{7}$ Petersburg Nuclear Physics Institute, 188300 St. Petersburg, Russia.
}

\begin{abstract}
The full counting statistics for the charge transport through an undoped graphene sheet in the presence of strong potential impurities is studied. Treating the scattering off the impurity in the $s$ wave approximation, we calculate the impurity correction to the cumulant generating function. This correction is universal provided the impurity strength is tuned to a resonant value. In particular, the conductance of the sample acquires a correction of $16 e^{2} /\left(\pi^{2} h\right)$ per resonant impurity.
\end{abstract}

PACS numbers: 73.63.-b, 73.22.-f

Since the discovery of graphene [1] its transport properties have become a subject of intense studies [2, 3]. The most remarkable effects arise when the chemical potential is tuned into a close vicinity of the Dirac point. In particular, a short and wide sample of clean graphene exhibits a pseudo-diffusive charge transport [4], with the counting statistics equivalent to that of a diffusive wire $[5,6]$. This equivalence has been confirmed in recent measurements of conductance and noise in ballistic graphene flakes [7, 8]. In contrast to conventional metals, ballistic graphene near the Dirac point conducts better when potential impurities are added [9 11]. Quantum interference in disordered graphene is also highly peculiar due to Dirac nature of carriers. In particular, in the absence of intervalley scattering, the minimal conductivity $\sim e^{2} / h$ [2] is "protected" from quantum localization [12].

Strong impurities creating resonances near the Dirac point ("midgap states") are one of the most plausible mechanisms limiting the electron mobility and can be used exploited for functionalization of graphene. As was shown in Refs. 13, 14], such scatterers provide the concentration dependence of the conductivity, $\sigma \propto n \ln ^{2} n$, which is consistent with most of experimental observations. Possible realizations of such scatterers are vacancies, adsorbed atoms, molecules, or impurity clusters [15, 16]. In particular, important example is hydrogen atoms that can be controllably added to the graphene sample [17]. Resonant scattering may also be intentionally induced by metallic islands deposited on graphene surface [18]. In this case the strength of impurity can be controlled by a local external gate.

In this paper we study the effect of strong impurities on electron transport in graphene. We consider the ballistic transport regime in which the sample size is smaller than the electron mean free path (low impurity concentration). This model was experimentally implemented in the samples on the $\mathrm{SiO}_{2}$ substrate [7, 8, 19]. The ballistic transport is particularly relevant for suspended samples

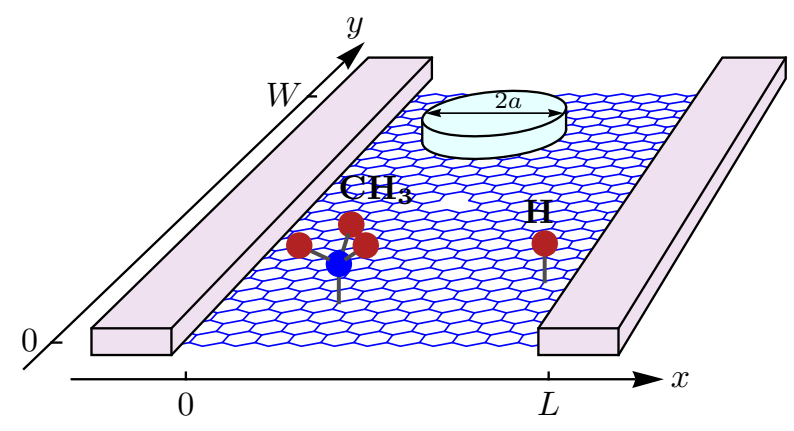

FIG. 1: (Color online) Ballistic graphene setup with various strong scatterers. Vacancies as well as atomic or molecular impurities can create midgap states [15]. Metallic islands support quasibound states that can be tuned to the resonance.

where much higher mobilities have been achieved [20, 21].

We evaluate analytically the effect of strong impurities on the full counting statistics (FCS) in a rectangular sample of length $L$ and width $W$ (Fig. (1). In particular, for the short-and-wide setup, $W \gg L$, we find the universal corrections to the conductance $G$ and to the shot noise $S$ (divided by $2 \mathrm{eV}$, where $V$ is the bias voltage),

$$
\delta G=\frac{16}{\pi^{2}} \frac{e^{2}}{h}, \quad \frac{\delta S}{2 e V}=\left(\frac{1}{3}-\frac{3}{\pi^{2}}\right) \frac{16}{\pi^{2}} \frac{e^{2}}{h},
$$

per resonant potential scatterer of the round shape.

We consider an effective model described by the Dirac Hamiltonian, $H=-i \hbar v \boldsymbol{\sigma} \nabla+U(\mathbf{r})$, where $\boldsymbol{\sigma}=\left(\sigma_{x}, \sigma_{y}\right)$ is the vector of Pauli matrices, $v$ is the velocity, and $U(\mathbf{r})$ is the impurity potential. Below, we set $\hbar v=1$. Two metallic leads at $x<0$ and $x>L$ are defined by adding a large chemical potential to the graphene Hamiltonian. Inside the sample, i.e. for $0<x<L$, the chemical potential is set to zero (Dirac point). The potential $U(\mathbf{r})$ represents a collection of randomly distributed sharp scatterers of arbitrary strength.

We will study FCS of ballistic electron transport 
through the sample using two complementary approaches - scattering theory and Green function formalism [22]. The first approach works for any aspect ratio of the sample while impurities are treated as point-like. The second method is particularly suitable for $W \gg L$ and allows for arbitrary impurity profile.

We begin with the scattering approach for a single impurity [9]. We discretize $y$ coordinate, $y=W \nu / N$, where $\nu=1,2, \ldots, N$ is an integer, $N=W / \pi a \gg 1$, and $a \ll L$ plays the role of the impurity size. The impurity potential in this model is defined as

$$
U(\mathbf{r})=\alpha \delta\left(x-x_{0}\right) \delta_{\nu \nu_{0}}
$$

where $x_{0}$ and $y_{0}=W \nu_{0} / N$ specify the impurity position. Generically, $\alpha$ is a $4 \times 4$ matrix in sublattice and valley spaces. Below we concentrate on the case of scalar potential with $\alpha$ being a number.

In order to study transport properties, we perform a standard unitary rotation 9 of the Hamiltonian, $H \mapsto$ $\mathcal{L}^{\dagger} H \mathcal{L}$ with $\mathcal{L}=\left(\sigma_{x}+\sigma_{z}\right) / \sqrt{2}$. After this transformation the upper (lower) element of the spinor wave function represents right- (left-) propagating mode in the leads. We then perform the discrete Fourier transform with respect to $\nu$ and arrive at the Dirac equation

$$
\frac{\partial \Psi(x)}{\partial x}=\left[\sigma_{x} \hat{q}-i \sigma_{z} \hat{U}(x)\right] \Psi(x)
$$

where $\hat{q}$ is a diagonal matrix with entries $q_{n}=$ $2 \pi n / W$ being the discrete transverse momenta and $n=$ $-N / 2, \ldots, N / 2$ (for definiteness, we assume periodic boundary conditions). The impurity potential is represented by the operator $\hat{U}(x)=\alpha \delta\left(x-x_{0}\right)|\Phi\rangle\langle\Phi|$ that projects onto the state with the wave function $\Phi\left(q_{n}\right)=$ $N^{-1 / 2} e^{i q_{n} y_{0}}$. Using this separable form of the impurity potential $\hat{U}$, we can explicitly calculate the transfer matrix $\mathcal{T}$ that relates the wave-function amplitudes in the opposite leads, $\Psi(L)=\mathcal{T} \Psi(0)$. The result is given by

$$
\mathcal{T}=e^{\sigma_{x} \hat{q}\left(L-x_{0}\right)} e^{i \sigma_{z} \alpha|\Phi\rangle\langle\Phi|} e^{\sigma_{x} \hat{q} x_{0}} .
$$

Inverting the element $\mathcal{T}_{11}$ (where indices refer to the $\sigma$ space), we obtain an exact expression for the transmission amplitude from the $n$th channel in the left lead to the $m$ th channel in the right lead (see Appendix $\mathrm{A}$ ),

$$
t_{n m}=\frac{\delta_{n m}}{\cosh q_{n} L}-\frac{2 \gamma_{n m}[z+i N \cot (\alpha / 2)]^{-1}}{\cosh q_{n} L \cosh q_{m} L},
$$

with $\gamma_{n m}=e^{i\left(q_{n}-q_{m}\right) y_{0}} \cosh \left[q_{n} x_{0}-q_{m}\left(L-x_{0}\right)\right]$ and

$$
z=\sum_{n} \cosh \left[q_{n}\left(L-2 x_{0}\right)\right] / \cosh \left(q_{n} L\right) .
$$

The first term in Eq. (3) describes the clean system $(\alpha=0)$. The second term represents the effect of the impurity which is particularly important in the vicinity

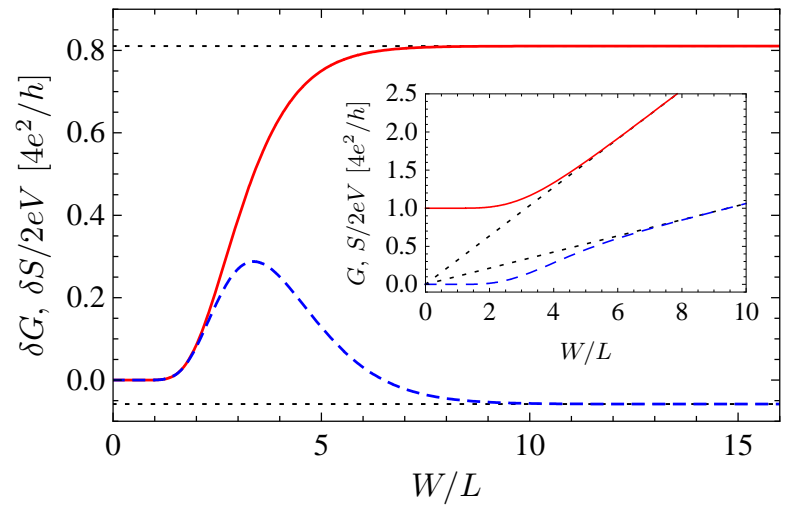

FIG. 2: (Color online) Contribution to the conductance $G$ (solid) and to the shot noise $S$ (dashed) from a resonant impurity placed at $x_{0}=L / 2$, as a function of the aspect ratio $W / L$. Dotted lines show asymptotic values from Eq. (1). Inset: conductance and noise of a clean sample, cf. Ref. [5].

of resonant values $\alpha_{n}=\pi(2 n+1)$. The quantity $z$ defined by Eq. (4) is $\pi W$ times the local density of states of a clean system at the position of impurity.

The statistics of the charge transport is described by the cumulant generating function $\mathcal{F}(\chi)$ [23] as $c_{n}=$ $\lim _{\chi \rightarrow 0} \partial^{n} \mathcal{F} / \partial \chi^{n}$, where $c_{1}=G / g_{0}$ is the dimensionless conductance, $c_{2}=S / 2 \mathrm{eVg}$ is the dimensionless noise, etc. For our purposes it is more convenient to use the variable $\phi$ defined via $\chi=2 \ln |\cos (\phi / 2)|$, which yields

$$
\mathcal{F}(\phi)=\ln \operatorname{det}\left[1-\hat{t} \hat{t}^{\dagger} \sin ^{2}(\phi / 2)\right] .
$$

Using Eq. (3), we calculate conductance and noise of the sample with a resonant impurity placed in the center, see Fig. 2. The general expression for the generating function applicable for any strength and position of the impurity is given in Appendix A.

In the limit $W \gg L$ the summations over transversal modes can be replaced by momentum integrals. For the clean system this yields $\mathcal{F}_{0}=-W \phi^{2} / 4 \pi L$. The impurity correction to $\mathcal{F}$ takes the form

$$
\delta \mathcal{F}=2 \ln \left[1-\frac{\ell^{2}}{(4 L)^{2}}\left(\frac{\phi^{2}}{\pi^{2}}-\frac{1}{\sin ^{2}\left(\pi x_{0} / L\right)}\right)\right],
$$

where $\ell=2 \pi a \tan (\alpha / 2)$ (as we show below, $\ell$ is the scattering length at low energies). In particular, the correction to the conductance $G_{0}=g_{0} W / \pi L$ reads

$$
\delta G=\left(8 g_{0} / \pi^{2}\right)\left[\sin ^{-2}\left(\pi x_{0} / L\right)+(4 L)^{2} / \ell^{2}\right]^{-1} .
$$

This completes our analysis of the discretized model (2).

Let us now turn to the microscopic description of the impurity within the continuous Dirac model, assuming that the impurity potential is rotational symmetric, $U(\mathbf{r})=u\left(\left|\mathbf{r}-\mathbf{r}_{0}\right|\right)$, where $\mathbf{r}_{0}$ is the impurity position. To calculate FCS we use the matrix Green function approach 
[6, 22]. The Green function in the retarded-advanced (RA) space satisfies the equation

$$
\left(\begin{array}{cc}
\mu(x)-H+i 0 & -\sigma_{x} \zeta \delta(x) \\
-\sigma_{x} \zeta \delta(x-L) & \mu(x)-H-i 0
\end{array}\right) \check{G}\left(\mathbf{r}, \mathbf{r}^{\prime}\right)=\delta\left(\mathbf{r}-\mathbf{r}^{\prime}\right),
$$

where $\zeta=\sin (\phi / 2)$ is the counting field and $\mu$ is the chemical potential which is zero inside the sample and infinite in the leads. An explicit solution to the above equation is given in Appendix B.

The generating function can be expressed through $\check{G}$ as $\mathcal{F}(\phi)=\operatorname{Tr} \ln \mathscr{G}^{-1}$, where $\operatorname{Tr}$ is the full operator trace. Expanding $\mathcal{F}(\phi)$ in $U(\mathbf{r})$, we obtain the impurity correction to the generating function as a series

$$
\delta \mathcal{F}=-\sum_{p=1}^{\infty} \frac{1}{p} \operatorname{Tr}\left(U \check{G}_{0}\right)^{p}
$$

where $\check{G}_{0}$ is the Green function of the clean system. Evaluation of Eq. (8) essentially simplifies if the impurity size $a$ is small compared to $L$. In this limit the arguments of the Green function are close to the position of the impurity, $\mathbf{r}_{0}$. Therefore it is useful to split $\breve{G}_{0}$ into the singular and regular parts, $\breve{G}_{0}=g+\breve{G}_{\text {reg }}$, where

$$
g\left(\mathbf{r}, \mathbf{r}^{\prime}\right)=-(i / 2 \pi) \boldsymbol{\sigma} \cdot\left(\mathbf{r}-\mathbf{r}^{\prime}\right) /\left|\mathbf{r}-\mathbf{r}^{\prime}\right|^{2}
$$

is the zero-energy Green function of the free Dirac fermion. The regular part of the Green function, $\check{G}_{\text {reg, }}$, can be taken with equal arguments $\mathbf{r}=\mathbf{r}^{\prime}=\mathbf{r}_{0}$. Explicit expression for $\check{G}_{\text {reg }}(\mathbf{r})$ is given in Appendix B. We characterize the impurity by its $T$-matrix

$$
T=\sum_{p=1}^{\infty} \int d^{2} \mathbf{r}_{1} \ldots d^{2} \mathbf{r}_{p} U\left(\mathbf{r}_{1}\right) g\left(\mathbf{r}_{1}, \mathbf{r}_{2}\right) \ldots U\left(\mathbf{r}_{p}\right)
$$

Rearranging singular and regular parts in Eq. (8) yields

$$
\delta \mathcal{F}=\operatorname{Tr} \ln \left[1-T \check{G}_{\mathrm{reg}}\left(\mathbf{r}_{0}\right)\right] .
$$

Note that this result holds for any $T$ matrix, including possible valley mixing. In the case of rotational invariant impurity, the $T$ matrix reduces to the scattering length $\ell$ in the $s$-channel. Since $\operatorname{Tr} \breve{G}_{\text {reg }}\left(\mathbf{r}_{0}\right)=0$ and $\check{G}_{\text {reg }}^{2}\left(\mathbf{r}_{0}\right)=\left[\phi^{2} / \pi^{2}-\sin ^{-2}\left(\pi x_{0} / L\right)\right] /(4 L)^{2}$ (Appendix B), the impurity correction to the generating function reproduces the result (6). This establishes a relation between the phenomenological parameter $\alpha$ in Eq. (2) and the actual profile of the impurity potential.

It is convenient to derive the scattering length $\ell$ from the solution of the Dirac equation in an infinite system [24] at a low energy $k$. Specifically, $\ell$ is related to the scattering cross-section $\Lambda$ as $\Lambda=k \ell^{2} / 2$. As an example, let us calculate $\ell$ for an impurity with the rectangular profile $u(r)=u_{0} \theta(a-r)$. Matching the solutions of the free Dirac equation at $r<a$ and $r>a$, we obtain

$$
\ell=2 \pi a J_{1}\left(u_{0} a\right) / J_{0}\left(u_{0} a\right),
$$

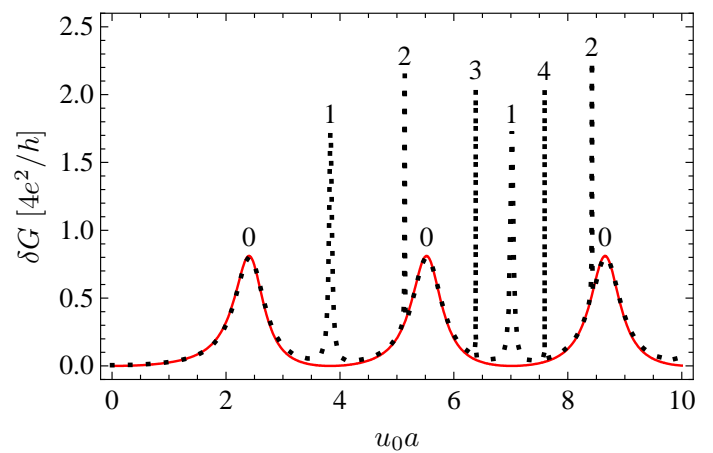

FIG. 3: (Color online) Correction to the conductance from a circular impurity at $x_{0}=L / 2$ as a function of $u_{0} a$. Solid line: analytic result, Eqs. (7), (9); dotted line: numerical simulations [25] with the parameters $a / L=0.2, W / L=6$.

where $J_{n}$ stands for the Bessel function. The scattering length diverges when the impurity potential develops a quasibound state (one component of the spinor wave function is localized) at zero energy. This implies resonant scattering, which is a generic feature of any strong impurity independent of its profile. In the quasiclassical limit, $u_{0} a \gg 1$, one finds $\ell=2 \pi a \tan \left(u_{0} a-\pi / 4\right)$, which corresponds to $\alpha=2 u_{0} a-\pi / 2$ in Eq. (2).

In Fig. 3 we compare the results (7), (9) to the conductance calculated numerically in Ref. [25] for the case of the disk impurity. In addition to the $s$-wave resonances, that are perfectly described by our theory, there exist higher resonances that can be included into our consideration in a similar manner, see Appendix C] The position of the resonances are given by the zeroes of $J_{m}\left(u_{0} a\right)$, where the corresponding index $m=0,1,2, \ldots$ is specified in Fig. 3. The resonance widths scale as $(a / L)^{2 m+1}$.

Now we turn to the case of disordered graphene with a small concentration of impurities randomly distributed over the sample. The average generating function $\overline{\mathcal{F}}$ can be found to the linear order in $n_{\text {imp }}$ (see Fig. 4 a) by the integration of Eq. (6) over the sample area,

$$
\overline{\mathcal{F}}=-\frac{W \phi^{2}}{4 \pi L}+4 N_{\mathrm{imp}} \ln \left(1+\sqrt{1+\frac{16 L^{2}}{\ell^{2}}-\frac{\phi^{2}}{\pi^{2}}}\right),
$$

where $N_{\mathrm{imp}}=n_{\mathrm{imp}} W L$ is the total number of impurities. Resonant impurities $(\ell=\infty)$ contribute on average the universal correction (11) to the conductance and noise, yielding the conductivity $\sigma=G L / W$ and Fano factor

$$
\sigma=\frac{4 e^{2}}{\pi h}\left(1+\frac{4}{\pi} n_{\mathrm{imp}} L^{2}\right), \quad F=\frac{1}{3}-\frac{12}{\pi^{3}} n_{\mathrm{imp}} L^{2} .
$$

Away from a resonance, $a \ll \ell \ll L$, we find

$$
\sigma=\frac{4 e^{2}}{\pi h}\left(1+\frac{n_{\mathrm{imp}} \ell^{2}}{2 \pi}\right), \quad F=\frac{1}{3}-\frac{n_{\mathrm{imp}} \ell^{4}}{8 \pi^{3} L^{2}} .
$$

To establish the limits of validity of these ballistic results, we estimate the contribution of the order $n_{\mathrm{imp}}^{2}$ in 


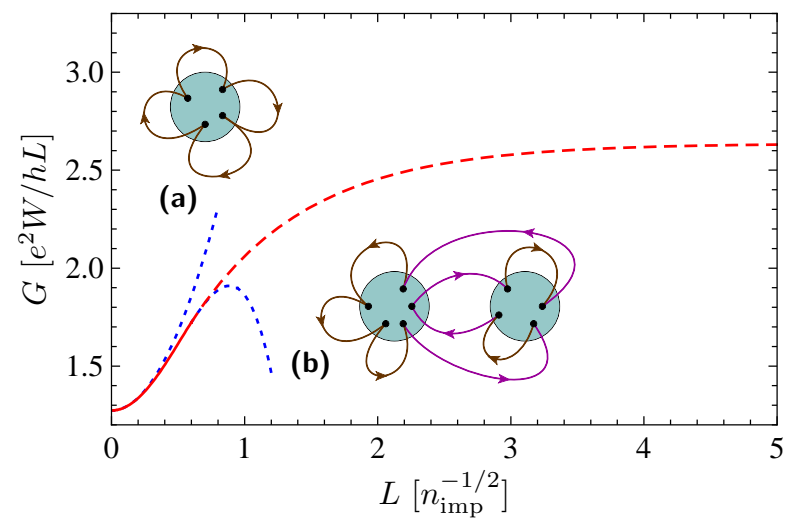

FIG. 4: (Color online) Conductance vs. $L$ for resonant impurities. Dotted lines: (a) one- and (b) two-impurity contributions. Inset: corresponding typical diagrams. Dashed line illustrates schematically the crossover to diffusive regime.

the virial expansion. The diagrams involving two resonant impurities, Fig. 迎, yield the free-energy correction

$\delta^{(2)} \mathcal{F}=\frac{1}{2} \operatorname{Tr} \ln \left[1-\check{G}_{\mathrm{reg}}^{-1}\left(\mathbf{r}_{1}\right) \check{G}_{0}\left(\mathbf{r}_{1}, \mathbf{r}_{2}\right) \check{G}_{\mathrm{reg}}^{-1}\left(\mathbf{r}_{2}\right) \check{G}_{0}\left(\mathbf{r}_{2}, \mathbf{r}_{1}\right)\right]$,

which is a contribution of atypical pairs of scatterers separated by a distance $\lesssim L$. The corresponding corrections to the conductance and Fano factor are (Appendix D)

$$
\delta^{(2)} \sigma=-1.032 \frac{e^{2}}{h} n_{\mathrm{imp}}^{2} L^{4}, \quad \delta^{(2)} F=0.8623 n_{\mathrm{imp}}^{2} L^{4} .
$$

Two closely located strong impurities detune each other from the resonance, hence the negative correction to the conductance. For off-resonance impurities, $\ell \ll L$, the multiple scattering correction to conductance can be derived from the first-order result Eq. (10) supplied by the logarithmic renormalization of the effective impurity strength $\alpha_{0}=n_{\text {imp }} \ell^{2} / 2 \pi$ according to Refs. [10, 13],

$$
\sigma=\frac{4 e^{2}}{\pi h}\left(1+\frac{n_{\mathrm{imp}} \ell^{2}}{2\left[\pi-n_{\mathrm{imp}} \ell^{2} \log (L / \ell)\right]}\right) .
$$

When $\delta \sigma \sim e^{2} / h$ (which corresponds to mean free path $\sim L$ ), the system enters the diffusive regime, Fig. 4. This happens at $n_{\text {imp }} \min \left\{L^{2}, \ell^{2}|\log (L / \ell)|\right\} \sim 1$. Remarkably, for resonant impurities this implies just one impurity per square $L \times L$. The scaling of conductivity in the diffusive regime is determined by the symmetries of impurities in sublattice and valley space [12]. In particular, for vacancies the quantum interference is suppressed in view of chiral symmetry [13]. This should yield the universal (as long as $N_{\mathrm{imp}}$ is small compared to the number of carbon atoms) minimal conductivity $\sim e^{2} / h$.

The situation with strong potential scatterers (e.g., adsorbants inducing midgap states) is similar for intermediate $L$ where the chiral symmetry is approximately preserved, Fig. 4. At larger scales, $L>L_{c}$, violation of this symmetry becomes important, and quantum interference comes into play. The symmetry-breaking length $L_{c}$ is given by $L_{c} \sim n_{\mathrm{imp}}^{-1 / 2} \min \left\{n_{\mathrm{imp}} \ell^{2}, 1 / n_{\mathrm{imp}} a^{2}\right\}$ : the chiral symmetry is broken due to detuning from resonance as well as due to higher (non-resonant) scattering channels. For impurities that do not mix the valleys (i.e. smooth on the scale of the lattice constant) this implies antilocalization (symplectic symmetry class) leading to logarithmic increase of conductivity with $L$. The valley mixing restores conventional (orthogonal) 2D localization.

In conclusion, we have computed FCS for charge transport through an undoped graphene sheet in the presence of strong potential impurities. The impurity correction to FCS, Eq. (6), is determined by the position of impurity and the low-energy scattering length. The latter diverges when the impurity potential has a quasibound state at zero energy. At such resonant conditions the impurity correction becomes universal, Eq. (1). Our results are fully supported by numerical simulations, Fig. 3 .

We are grateful to J. Bardarson and T. O. Wehling for valuable discussions. We thank Centro de Ciencias de Benasque, Spain where this work was completed. The work was supported by the EUROHORCS/ESF EURYI Award (I.V.G.) and Rosnauka grant 02.740.11.5072.

[1] K. S. Novoselov et al., Science 306, 666 (2004).

[2] K. S. Novoselov et al., Nature (London) 438, 197 (2005); Y.Zhang et al., Nature (London) 438, 201 (2005); Y.W. Tan et al., Eur. Phys. J. Spec. Top. 148, 15 (2007).

[3] A. H. Castro Neto et al., Rev. Mod. Phys. 81, 109 (2009).

[4] M. I. Katsnelson, Eur. Phys. J. B 51, 157 (2006).

[5] J. Tworzydło et al., Phys. Rev. Lett. 96, 246802 (2006); C. W. J. Beenakker, Rev. Mod. Phys. 80, 1337 (2008).

[6] S. Ryu et al., Phys. Rev. B 75, 205344 (2007).

[7] F. Miao, et al., Science 317, 1530 (2007).

[8] R. Danneau et al., Phys. Rev. Lett. 100, 196802 (2008).

[9] M. Titov, Europhys. Lett. 79, 17004 (2007).

[10] A. Schuessler et al., Phys. Rev. B 79, 075405 (2009).

[11] J. H. Bardarson, et al., Phys. Rev. Lett. 99, 106801 (2007); K. Nomura et al., ibid. 99, 146806 (2007); P.San-Jose et al., Phys. Rev. B 76, 195445 (2007); C. H. Lewenkopf et al., ibid. 77, 081410R (2008); J. Tworzydlo et al., ibid. 78, 235438 (2008).

[12] P. M. Ostrovsky et al., Phys. Rev. Lett. 98, 256801 (2007); Eur. Phys. J. Spec. Top. 148, 63 (2007).

[13] P. M. Ostrovsky et al., Phys. Rev. B 74, 235443 (2006).

[14] T. Stauber et al., Phys. Rev. B 76, 205423 (2007).

[15] T. O. Wehling et al., Phys. Rev. B 75, 125425 (2007).

[16] F. Schedin et al., Nat. Mater. 6, 652 (2007).

[17] D. C. Elias et al., Science 323, 610 (2009).

[18] B. M. Kessler et al., arXiv:0907.3661.

[19] H. B. Heersche et al., Nature 446, 56 (2007).

[20] K. I. Bolotin et al., Solid State Commun. 146, 351 (2008).

[21] Xu Du et al., Int. J. Mod. Phys. B 22, 4579 (2008).

[22] Yu. V. Nazarov, Phys. Rev. Lett. 73, 134 (1994).

[23] L.S.Levitov and G.B.Lesovik, JETP Lett. 58, 230 (1993).

[24] M. Hentschel and F. Guinea, Phys. Rev. B 76, 115407 (2007); D. S. Novikov, ibid. 76, 245435 (2007); D. M. Basko, ibid. 78, 115432 (2008).

[25] J. Bardarson et al., Phys. Rev. Lett. 102, 226803 (2009). 


\section{Appendix A: Transfer matrix approach}

The full counting statistics of the charge transport is encoded in the cumulant generating function given by the formula

$$
\mathcal{F}(\phi)=\ln \operatorname{det}\left[1-\hat{t} \hat{t}^{\dagger} \sin ^{2}(\phi / 2)\right]
$$

where $\hat{t}$ is an $N \times N$ matrix of transmission amplitudes, which are defined with respect to the scattering states in the leads. The matrix $\hat{t}$ is obtained by inverting the block $\mathcal{T}_{11}$ of the transfer matrix $\mathcal{T}$.

For the case of a single scalar impurity, the $2 N \times 2 N$ transfer matrix is given by the product of three matrix exponents [9]

$$
\mathcal{T}=e^{\sigma_{x} \hat{q}\left(L-x_{0}\right)} e^{i \alpha \sigma_{z}|\Phi\rangle\langle\Phi|} e^{\sigma_{x} \hat{q} x_{0}},
$$

where the coordinate $x_{0}$ specifies the position of the impurity in $x$ direction. The expression (A2) relays upon the sharp boundary conditions at the metal-graphene interfaces: $x=0$ and $x=L$. For periodic boundary conditions in $y$, the momentum quantization gives $q_{n}=\pi(2 n-N-1) / W$, where $n=1,2, \ldots, N$ (for simplicity, the number of channels, $N$, is taken to be an odd number), and the matrix $\hat{q}$ is defined as $\hat{q}=\operatorname{diag}\left(q_{1}, q_{2}, q_{3}, \ldots, q_{N}\right)$. It is already assumed in the construction of Eq. (A2) that the impurity is point-like in $x$ direction. Furthermore, if the impurity is point-like in $y$ direction the vector $|\Phi\rangle$ has the elements $\Phi\left(q_{n}\right)=N^{-1 / 2} e^{i q_{n} y_{0}}$, where $y_{0}$ specifies the impurity position in $y$ direction. The impurity strength in this phenomenological model is characterized by the dimensionless parameter $\alpha$.

In the limit of point-like impurity one can use the projective property of the operator $|\Phi\rangle\langle\Phi|$ in order to write

$$
e^{i \alpha \sigma_{z}|\Phi\rangle\langle\Phi|}=1-\left(1-e^{i \alpha \sigma_{z}}\right)|\Phi\rangle\langle\Phi| .
$$

Then the expression (A2) for the transfer matrix can be recast in the following form:

$$
\mathcal{T}=e^{\sigma_{x} \hat{q} L}-e^{\sigma_{x} \hat{q}\left(L-x_{0}\right)} D|\Phi\rangle\langle\Phi| e^{\sigma_{x} \hat{q} x_{0}},
$$

where we have introduced the abbreviation $D=1-\exp \left(i \alpha \sigma_{z}\right)$. It is also convenient to take advantage of $2 \times N$ matrix

$$
W_{x}=\frac{1}{\sqrt{N}}\left(\begin{array}{lllll}
\cosh q_{1} x & \cosh q_{2} x & \cosh q_{3} x & \ldots & \cosh q_{N} x \\
\sinh q_{1} x & \sinh q_{2} x & \sinh q_{3} x & \ldots & \sinh q_{N} x
\end{array}\right) e^{i \hat{q} y_{0}}
$$

which helps rewriting the matrix of transmission amplitudes, $\hat{t}=\left(\mathcal{T}_{11}\right)^{-1}$, as

$$
\hat{t}=\frac{1}{\cosh \hat{q} L-W_{L-x_{0}}^{\dagger} D W_{x_{0}}}=\frac{1}{\cosh \hat{q} L}+\frac{1}{\cosh \hat{q} L} W_{L-x_{0}}^{\dagger} \frac{1}{D^{-1}-W_{x_{0}} \frac{1}{\cosh \hat{q} L} W_{L-x_{0}}^{\dagger}} W_{x_{0}} \frac{1}{\cosh \hat{q} L} .
$$

Thus the inversion of $N \times N$ block of the transfer matrix is reduced to the inversion of $2 \times 2$ matrix in the last expression. Indeed, the straightforward calculation yields

$$
W_{x_{0}} \frac{1}{\cosh \hat{q} L} W_{L-x_{0}}^{\dagger}=\frac{1}{2}\left(1+\frac{z}{N} \sigma_{z}\right), \quad z=\sum_{n} \frac{\cosh q_{n}\left(L-2 x_{0}\right)}{\cosh q_{n} L} .
$$

Substituting this result into Eq. (A6) we obtain

$$
\hat{t}=\frac{1}{\cosh \hat{q} L}-\frac{1}{\cosh \hat{q} L} W_{L-x_{0}}^{\dagger} K W_{x_{0}} \frac{1}{\cosh \hat{q} L}, \quad K=\frac{2}{1+(z / N) \sigma_{z}-2 D^{-1}}=\frac{2 N \sigma_{z}}{z-i N \cot (\alpha / 2)} .
$$

This expression is equivalent to Eq. (3). Using Eq. (A8) one can write the matrix product $\hat{t}^{\dagger} \hat{t}$ as

$$
\hat{t}^{\dagger} \hat{t}=\frac{1}{\cosh \hat{q} L}\left(1+\tilde{W}^{\dagger} \Sigma \tilde{W}\right) \frac{1}{\cosh \hat{q} L}
$$

where $\tilde{W}$ is a rectangular matrix of the dimension $4 \times N$ and $4 \times 4$ matrix $\Sigma$ is responsible for the impurity correction to the transmission probabilities,

$$
\tilde{W}=\left(\begin{array}{c}
W_{x_{0}} \\
W_{L-x_{0}} \frac{1}{\cosh \hat{q} L}
\end{array}\right), \quad \Sigma=\left(\begin{array}{cc}
K^{\dagger} W_{L-x_{0}} \frac{1}{\cosh ^{2} \hat{q} L} W_{L-x_{0}}^{\dagger} K-K^{\dagger} \\
-K & 0
\end{array}\right) .
$$


Using the decomposition (A9) we can essentially simplify the calculation of the determinant in Eq. (A1) for the full counting statistics. First, we factorize the determinant into the product of two determinants: one for the full counting statistics of a clean system and the other one for the impurity correction,

$$
e^{\mathcal{F}}=\operatorname{det}\left(1-\frac{\sin ^{2}(\phi / 2)}{\cosh ^{2} \hat{q} L}\right) \operatorname{det}\left(1-\frac{\sin ^{2}(\phi / 2)}{\cosh ^{2} \hat{q} L-\sin ^{2}(\phi / 2)} \tilde{W}^{\dagger} \Sigma \tilde{W}\right) .
$$

The first determinant of the diagonal matrix is evaluated straightforwardly. In the second one we perform a cyclic permutation of matrices and reduce it from $N \times N$ down to $4 \times 4$. This matrix is diagonal in $\sigma$ space and hence the determinant further reduces to the product of $2 \times 2$ determinants. After some algebraic manipulations we find out that these two latter determinants are identical. This allows us to represent the generating function $\mathcal{F}$ as a sum $\mathcal{F}=\mathcal{F}_{0}+\delta \mathcal{F}$, where $\mathcal{F}_{0}$ describes the clean system and $\delta \mathcal{F}$ provides the impurity contribution to the full counting statistics,

$$
\mathcal{F}_{0}=\sum_{n} \ln \left[1-\frac{\sin ^{2}(\phi / 2)}{\cosh ^{2} q_{n} L}\right], \quad \delta \mathcal{F}=2 \ln \left[1+\frac{P(\phi)-R_{0}^{2} \sin ^{2} \phi}{z^{2}+N^{2} \cot ^{2}(\alpha / 2)}\right]
$$

Here we have introduced the following notations:

$$
R_{x}=\frac{1}{2} \sum_{n} \frac{\cosh \left(2 q_{n} x\right)}{\cosh ^{2}\left(q_{n} L\right)-\sin ^{2}(\phi / 2)}, \quad P(\phi)=R_{L-x_{0}}^{2}+2 R_{L-x_{0}} R_{x_{0}} \cos \phi+R_{x_{0}}^{2}-z^{2} .
$$

The generating function $\mathcal{F}=\mathcal{F}_{0}+\delta \mathcal{F}$ given by Eq. (A12) is a general solution of the single-impurity problem applicable for any strength and position of the impurity and arbitrary aspect ratio of the sample.

In the limit $W \gg L$ the summations over transversal modes can be replaced by momentum integrals, so that

$$
R_{x}=\frac{W}{2 L} \frac{\sin (\phi x / L)}{\sin (\pi x / L) \sin \phi}, \quad R_{0}=\frac{W \phi}{2 \pi L \sin \phi}, \quad z=\frac{W}{2 L \sin \left(\pi x_{0} / L\right)}, \quad P(\phi)=0 .
$$

As a result, one obtains Eq. (6).

\section{Appendix B: Matrix Green function}

The full counting statistics of the electron transport is conveniently expressed in terms of the matrix Green function 22] in the external counting field $\zeta=\sin (\phi / 2)$. For the clean graphene sample this Green function satisfies the following equation in the retarded-advanced space:

$$
\left(\begin{array}{cc}
\mu(x)-\boldsymbol{\sigma} \mathbf{p}+i 0 & -\sigma_{x} \zeta \delta(x) \\
-\sigma_{x} \zeta \delta(x-L) & \mu(x)-\boldsymbol{\sigma} \mathbf{p}-i 0
\end{array}\right) \check{G}_{0}\left(\mathbf{r}, \mathbf{r}^{\prime}\right)=\delta\left(\mathbf{r}-\mathbf{r}^{\prime}\right), \quad \mu(x)= \begin{cases}0, & 0<x<L, \\
+\infty, & x<0 \text { or } x>L\end{cases}
$$

Since the operator in the left-hand side of the above equation commutes with the $y$ component of the momentum, we will first calculate the Green function in the mixed coordinate-momentum representation, $\check{G}_{p}\left(x, x^{\prime}\right)$. Inside the sample this function satisfies

$$
\left[i \sigma_{x} \frac{\partial}{\partial x}-\sigma_{y} p\right] \check{G}_{p}\left(x, x^{\prime}\right)=\delta\left(x-x^{\prime}\right)
$$

We will look for a general solution of this equation in the form

$$
\check{G}_{p}\left(x, x^{\prime}\right)=e^{\sigma_{z} p(x-L / 2)} M e^{\sigma_{z} p\left(x^{\prime}-L / 2\right)}, \quad M= \begin{cases}M_{<}, & x<x^{\prime}, \\ M_{>}, & x>x^{\prime}\end{cases}
$$

The chemical potential profile together with the infinitesimal terms $\pm i 0$ in Eq. (B1) defines the boundary conditions for the Green function. The counting field $\zeta$ can also be incorporated into the boundary conditions. In terms of $M_{\lessgtr}$ we thus obtain

$$
\left(\begin{array}{cccc}
1 & 1 & i \zeta & i \zeta \\
0 & 0 & 1 & -1
\end{array}\right) e^{-\sigma_{z} p L / 2} M_{<}=0, \quad\left(\begin{array}{cccc}
1 & -1 & 0 & 0 \\
-i \zeta & -i \zeta & 1 & 1
\end{array}\right) e^{\sigma_{z} p L / 2} M_{>}=0
$$


Delta function in the right-hand side of Eq. (B2) yields a jump of the Green function at $x=x^{\prime}$ which provides the relation

$$
M_{>}-M_{<}=-i \sigma_{x}
$$

The matrices $M_{\lessgtr}$, and hence the Green function, are completely determined by Eqs. (B4, (B5),

$$
M_{\lessgtr}=\frac{-i}{2\left(\cosh ^{2} p L-\zeta^{2}\right)}\left(\begin{array}{cccc}
\cosh p L & \zeta^{2}-\frac{\sinh 2 p L}{2} & i \zeta e^{-p L} & i \zeta \\
\zeta^{2}+\frac{\sinh 2 p L}{2} & \cosh p L & i \zeta & i \zeta e^{p L} \\
i \zeta e^{p L} & i \zeta & -\cosh p L & -\zeta^{2}-\frac{\sinh 2 p L}{2} \\
i \zeta & i \zeta e^{-p L} & -\zeta^{2}+\frac{\sinh 2 p L}{2} & -\cosh p L
\end{array}\right) \pm \frac{i \sigma_{x}}{2} .
$$

Fourier transform in $p$ yields the Green function in the full coordinate representation. To facilitate further calculations, we decompose this Green function into the following product of matrices:

$$
\begin{array}{r}
\check{G}_{0}\left(x, x^{\prime} ; y\right)=\frac{1}{4} \check{V}(x) \check{\Lambda}\left(\begin{array}{cc}
i \cosh \frac{\phi y}{2 L} & \sinh \frac{\phi y}{2 L} \\
\sinh \frac{\phi y}{2 L} & -i \cosh \frac{\phi y}{2 L}
\end{array}\right)_{R A}\left(\begin{array}{cc}
\frac{1}{\sin \frac{\pi}{2 L}\left(x+x^{\prime}+i y\right)} & \frac{1}{\sin \frac{\pi}{2 L}\left(x-x^{\prime}+i y\right)} \\
\frac{1}{\sin \frac{\pi}{2 L}\left(x-x^{\prime}-i y\right)} & \left.\frac{1}{\sin \frac{\pi}{2 L}\left(x+x^{\prime}-i y\right)}\right)_{\sigma}^{-1}\left(x^{\prime}\right), \\
\check{\Lambda}=\left(\begin{array}{cc}
\sigma_{z} & 0 \\
0 & 1
\end{array}\right), \quad \check{V}(x)=\left(\begin{array}{cc}
\sin \frac{\phi(L-x)}{2 L} & \cos \frac{\phi(L-x)}{2 L} \\
i \cos \frac{\phi x}{2 L} & i \sin \frac{\phi x}{2 L}
\end{array}\right)_{R A}
\end{array}\right.
\end{array}
$$

Here we have introduced the source angle $\phi$ defined by $\zeta=\sin (\phi / 2)$. The matrices $\check{V}(x)$ and $\check{V}^{-1}\left(x^{\prime}\right)$ operate in the retarded-advanced space only and hence commute with any disorder operators placed between the Green functions. As a result, factors $\check{V}$ and $\check{V}^{-1}$ drop from expressions for any closed diagrams. The matrices $\check{\Lambda}$ in the above equation allow us to decompose the Green function into a direct product of the two operators acting in the RA space and in the sublattice space. The matrix $\check{\Lambda}$ commutes only with the potential disorder and must be retained as a part of the Green function in the general case.

The regularized Green function arising in the calculation of diagrams with point-like impurities takes especially simple form when $\check{V}(x)$ and $\check{V}^{-1}(x)$ are singled out,

$$
\check{G}_{\mathrm{reg}}(x)=\lim _{\substack{x^{\prime} \rightarrow x \\
y \rightarrow 0}}\left[\check{G}\left(x, x^{\prime} ; y\right)+\frac{i}{2 \pi} \frac{\sigma_{x}\left(x-x^{\prime}\right)+\sigma_{y} y}{\left(x-x^{\prime}\right)^{2}+y^{2}}\right]=\frac{i}{4} \check{V}(x)\left(\begin{array}{cc}
\frac{1}{\sin \frac{\pi x}{L}} & -\sigma_{x} \frac{\phi}{\pi} \\
\sigma_{x} \frac{\phi}{\pi} & -\frac{1}{\sin \frac{\pi x}{L}}
\end{array}\right) \check{V}^{-1}(x) .
$$

It is worth noting that the above result does not depend on the order of taking the limits.

\section{Appendix C: Resonances in $p$-wave scattering}

In order to include higher scattering resonances in our consideration, we take into account small deviations of the regularized Green function endpoints from the center of impurity. Expansion up to the first order in these deviations allows for the resonances in the $p$ scattering channel. Let us define the following matrix containing the regularized Green function and its various derivatives:

$$
\check{\mathbb{G}}_{\mathrm{reg}}\left(\mathbf{r}_{0}\right)=\lim _{\substack{\mathbf{r} \rightarrow \mathbf{r}_{0} \\
\mathbf{r}^{\prime} \rightarrow \mathbf{r}_{0}}}\left[\begin{array}{ccc}
1 & \partial_{x^{\prime}} & \partial_{y^{\prime}} \\
\partial_{x} & \partial_{x} \partial_{x^{\prime}} & \partial_{x} \partial_{y^{\prime}} \\
\partial_{y} & \partial_{y} \partial_{x^{\prime}} & \partial_{y} \partial_{y^{\prime}}
\end{array}\right]\left[\check{G}_{0}\left(\mathbf{r}, \mathbf{r}^{\prime}\right)-g\left(\mathbf{r}-\mathbf{r}^{\prime}\right)\right] .
$$

Here and below we use the matrix notation with brackets rather than parentheses to distinguish the newly introduced matrix from the internal structure of the Green function in the RA and sublattice spaces. The [1,1] component of $\check{\mathbb{G}}_{\text {reg }}$ coincides with the regularized Green function $\check{G}_{\text {reg }}$ from Eq. (B9). 
The impurity is described by its $T$ matrix which is an operator with the following kernel:

$$
\begin{aligned}
& T\left(\mathbf{r}, \mathbf{r}^{\prime}\right)=U(\mathbf{r}) \delta^{(2)}\left(\mathbf{r}-\mathbf{r}^{\prime}\right)+U(\mathbf{r}) g\left(\mathbf{r}-\mathbf{r}^{\prime}\right) U\left(\mathbf{r}^{\prime}\right) \\
& \quad+\sum_{p=1}^{\infty} \int d^{2} \mathbf{r}_{1} \ldots d^{2} \mathbf{r}_{p} U(\mathbf{r}) g\left(\mathbf{r}-\mathbf{r}_{1}\right) U\left(\mathbf{r}_{1}\right) g\left(\mathbf{r}_{1}-\mathbf{r}_{2}\right) \ldots U\left(\mathbf{r}_{p}\right) g\left(\mathbf{r}_{p}-\mathbf{r}^{\prime}\right) U\left(\mathbf{r}^{\prime}\right) .
\end{aligned}
$$

In the main text we have focused on the $s$-wave resonances and characterized the impurity by its integrated $T$ matrix. In the simplest case of circular potential impurity the integrated $T$ matrix is nothing but the scattering length $\ell$ in the $s$ channel. Here we retain more information about the shape of the impurity and introduce the following integral of the $T$ matrix:

$$
\mathbb{T}=\int d^{2} \mathbf{r} d^{2} \mathbf{r}^{\prime}\left[\begin{array}{ccc}
1 & x^{\prime}-x_{0} & y^{\prime}-y_{0} \\
x-x_{0} & \left(x-x_{0}\right)\left(x^{\prime}-x_{0}\right) & \left(x-x_{0}\right)\left(y^{\prime}-y_{0}\right) \\
y-y_{0} & \left(y-y_{0}\right)\left(x^{\prime}-x_{0}\right) & \left(y-y_{0}\right)\left(y^{\prime}-y_{0}\right)
\end{array}\right] T\left(\mathbf{r}, \mathbf{r}^{\prime}\right) .
$$

Here and below we assume $\mathbf{r}=\{x, y\}$ and $\mathbf{r}^{\prime}=\left\{x^{\prime}, y^{\prime}\right\}$. The $[1,1]$ component of $\mathbb{T}$ is the integrated $T$ matrix used in the main text.

With the above-defined matrices $\check{\mathbb{G}}_{\text {reg }}$ and $\mathbb{T}$, we can express the single-impurity correction to the free energy in the form

$$
\delta \mathcal{F}=\ln \operatorname{det}\left[1-\mathbb{T} \check{G}_{\text {reg }}\left(\mathbf{r}_{0}\right)\right] .
$$

This expression includes the product of $[1,1]$ components of $\breve{G}_{\text {reg }}$ and $\mathbb{T}$, reproducing the result obtained in the main text, along with the next terms of the Taylor expansion of $\check{G}\left(\mathbf{r}, \mathbf{r}^{\prime}\right)$ in the vicinity of $\mathbf{r}_{0}$.

Let us now calculate the matrix $\breve{G}_{\text {reg }}$. Taking derivatives of $\breve{G}\left(\mathbf{r}, \mathbf{r}^{\prime}\right)$ we reveal the following internal structure of $\check{\mathbb{G}}_{\text {reg }}$ :

$$
\check{\mathbb{G}}_{\mathrm{reg}}(x)=\left[\begin{array}{cc}
1 & 0 \\
0 & \sigma_{y} \\
0 & \sigma_{x}
\end{array}\right]\left[\begin{array}{ll}
\check{G}_{\mathrm{reg}}(x) & \check{G}_{\mathrm{reg}}^{v}(x) \\
\check{G}_{\mathrm{reg}}^{v}(x) & \check{G}_{\mathrm{reg}}^{t}(x)
\end{array}\right]\left[\begin{array}{ccc}
1 & 0 & 0 \\
0 & \sigma_{y} & \sigma_{x}
\end{array}\right] .
$$

Here the element $\check{G}_{\text {reg }}$ is given by Eq. (B9) and the other elements are

$$
\begin{aligned}
& \check{G}_{\mathrm{reg}}^{v}(x)=\frac{\pi}{8 L} V(x)\left(\begin{array}{cc}
\sigma_{z} \frac{3 \phi^{2}-\pi^{2}}{6 \pi^{2}}-i \sigma_{y} \frac{\cos \frac{\pi x}{L}}{\sin ^{3} \frac{\pi x}{L}} & -i \sigma_{y} \frac{\phi}{\pi \sin \frac{\pi x}{L}} \\
-i \sigma_{y} \frac{\phi}{\pi \sin \frac{\pi x}{L}} & \sigma_{z} \frac{3 \phi^{2}-\pi^{2}}{6 \pi^{2}}+i \sigma_{y} \frac{\cos \frac{\pi x}{L}}{\sin ^{3} \frac{\pi x}{L}}
\end{array}\right) V^{-1}(x), \\
& \check{G}_{\mathrm{reg}}^{t}(x)=\frac{i}{8 L^{2}} V(x)\left(\begin{array}{cc}
\frac{\pi^{2}}{\sin ^{3} \frac{\pi x}{L}}-\frac{\pi^{2}+\phi^{2}}{2 \sin \frac{\pi x}{L}} & \frac{\pi \phi \cos \frac{\pi x}{L}}{\sin \frac{\pi x}{L}}-\sigma_{x} \phi \frac{\pi^{2}-\phi^{2}}{6 \pi} \\
\frac{\pi \phi \cos \frac{\pi x}{L}}{\sin \frac{\pi x}{L}}+\sigma_{x} \phi \frac{\pi^{2}-\phi^{2}}{6 \pi} & -\frac{\pi^{2}}{\sin ^{3} \frac{\pi x}{L}}+\frac{\pi^{2}+\phi^{2}}{2 \sin \frac{\pi x}{L}}
\end{array}\right) V^{-1}(x) .
\end{aligned}
$$

Taking the advantage of the representation (C5) we can reduce the size of the matrix in Eq. (C4) by introducing

$$
\mathbf{T}=\left[\begin{array}{ccc}
1 & 0 & 0 \\
0 & \sigma_{y} & \sigma_{x}
\end{array}\right] \mathbb{T}\left[\begin{array}{cc}
1 & 0 \\
0 & \sigma_{y} \\
0 & \sigma_{x}
\end{array}\right]=\int d^{2} \mathbf{r} d^{2} \mathbf{r}^{\prime}\left[\begin{array}{c}
1 \\
\sigma_{y}\left(x-x_{0}\right)+\sigma_{x}\left(y-y_{0}\right)
\end{array}\right] T\left(\mathbf{r}, \mathbf{r}^{\prime}\right)\left[\begin{array}{c}
1 \\
\sigma_{y}\left(x^{\prime}-x_{0}\right)+\sigma_{x}\left(y^{\prime}-y_{0}\right)
\end{array}\right]^{\dagger} .
$$

Now the correction to the free energy takes the simplified form

$$
\delta \mathcal{F}=\ln \operatorname{det}\left\{1-\mathbf{T}\left[\begin{array}{ll}
\check{G}_{\mathrm{reg}}\left(x_{0}\right) & \check{G}_{\mathrm{reg}}^{v}\left(x_{0}\right) \\
\check{G}_{\mathrm{reg}}^{v}\left(x_{0}\right) & \check{G}_{\text {reg }}^{t}\left(x_{0}\right)
\end{array}\right]\right\}
$$

In order to obtain the final result from Eq. (C9) we have to establish a relation between the matrix $\mathbf{T}$ and the solution of the scattering problem with a given impurity profile. Let us place the impurity at the origin $\mathbf{r}_{0}=0$ and consider the scattering state with the following asymptotic behavior far from the impurity:

$$
\psi(\mathbf{r})=\frac{e^{i \mathbf{k r}}}{\sqrt{2}}\left(\begin{array}{c}
1 \\
e^{i \varphi_{\mathbf{k}}}
\end{array}\right)-f_{k}\left(\varphi_{\mathbf{r}}, \varphi_{\mathbf{k}}\right) \sqrt{\frac{k}{4 \pi r}} e^{i k r+i \pi / 4}\left(\begin{array}{c}
1 \\
e^{i \varphi_{\mathbf{r}}}
\end{array}\right), \quad k r \gg 1 .
$$


This expression contains an incoming plane wave with the momentum $\mathbf{k}$ and an outgoing spherical wave with the direction-dependent scattering amplitude $f_{k}$. We use the notation $\varphi_{\mathbf{k}}\left(\varphi_{\mathbf{r}}\right)$ for the angle between the vector $\mathbf{k}(\mathbf{r})$ and the direction of $x$ axis.

The scattering amplitude is directly related to the matrix element of the $T$ matrix between the incoming and outgoing waves

$$
f_{k}\left(\varphi, \varphi^{\prime}\right)=\frac{1}{2} \int d^{2} \mathbf{r} d^{2} \mathbf{r}^{\prime}\left(1 e^{-i \varphi}\right) T\left(\mathbf{r}, \mathbf{r}^{\prime}\right)\left(\begin{array}{c}
1 \\
e^{i \varphi^{\prime}}
\end{array}\right) \exp \left[i k\left(x^{\prime} \cos \varphi^{\prime}+y^{\prime} \sin \varphi^{\prime}-x \cos \varphi-y \sin \varphi\right)\right] .
$$

The integrals entering the matrix $\mathbf{T}$ can be expressed through the $k \rightarrow 0$ limit of the scattering amplitude and its derivatives in the following way:

$$
\mathbf{T}=\lim _{k \rightarrow 0} \int_{0}^{2 \pi} \frac{d \varphi d \varphi^{\prime}}{2 \pi^{2}}\left(\begin{array}{cc}
1 & e^{-i \varphi^{\prime}} \\
e^{i \varphi} & e^{i\left(\varphi-\varphi^{\prime}\right)}
\end{array}\right)\left[\begin{array}{cc}
1 & -4 i \sin \left(2 \varphi^{\prime}\right) \frac{\partial}{\partial k} \\
4 i \sin (2 \varphi) \frac{\partial}{\partial k} & 8 \sin (2 \varphi) \sin \left(2 \varphi^{\prime}\right) \frac{\partial^{2}}{\partial k^{2}}
\end{array}\right] f_{k}\left(\varphi, \varphi^{\prime}\right) .
$$

Once the scattering amplitude for a given impurity is known, one can use Eqs. (C12) and (C9) to find the correction due to such an impurity to the full counting statistics, including $s$ - and $p$-wave scattering channels. Below we apply this general result to the circular potential impurity.

In the case of axially symmetric impurity, the scattering amplitude depends on the difference $\varphi-\varphi^{\prime}$ and Eq. (C12) simplifies to

$$
\mathbf{T}=\lim _{k \rightarrow 0} \int_{0}^{2 \pi} \frac{d \varphi}{\pi}\left(\begin{array}{cc}
1 & 0 \\
0 & e^{i \varphi}
\end{array}\right)\left[\begin{array}{cc}
1 & 0 \\
0 & 4 \cos (2 \varphi) \frac{\partial^{2}}{\partial k^{2}}
\end{array}\right] f_{k}(\varphi) .
$$

We can get rid of the $\varphi$ integral and express the result in terms of the scattering phases $\delta_{m}$ where $m$ is a half-integer number $-z$ component of the total momentum (angular + spin) of the Dirac electron. The scattering amplitude is the following Fourier series with the coefficients determined by the scattering phases:

$$
f_{k}(\varphi)=\frac{i}{k} \sum_{m}\left(e^{2 i \delta_{m}}-1\right) e^{i(m-1 / 2) \varphi} .
$$

In the case of potential impurity preserving the time-reversal invariance, the scattering phases obey the symmetry relation $\delta_{m}=\delta_{-m}$. At low energies they decay according to $\delta_{m}=O\left(k^{2|m|}\right)$. Using these properties, we substitute Eq. (C14) into Eq. (C13) and obtain

$$
\mathbf{T}=\left[\begin{array}{ll}
\ell & 0 \\
0 & \ell_{1}
\end{array}\right], \quad \ell=-4 \lim _{k \rightarrow 0} \frac{\delta_{1 / 2}}{k}, \quad \ell_{1}=-16 \lim _{k \rightarrow 0} \frac{\delta_{3 / 2}}{k^{3}} .
$$

Note that, contrary to $\ell$, the parameter $\ell_{1}$ has a dimension of the third power of length.

If the potential impurity has the rectangular profile with the height $u_{0}$ and radius $a$, the Dirac equation is easily solved yielding the following scattering parameters:

$$
\ell=2 \pi a \frac{J_{1}\left(u_{0} a\right)}{J_{0}\left(u_{0} a\right)}, \quad \ell_{1}=2 \pi a^{3} \frac{J_{2}\left(u_{0} a\right)}{J_{1}\left(u_{0} a\right)} .
$$

Now we evaluate the determinant in Eq. (C9) and obtain the result

$$
\begin{aligned}
\delta \mathcal{F}=2 \ln \left\{1-\frac{\ell^{2}}{16 L^{2}}\left(\frac{\phi^{2}}{\pi^{2}}-\frac{1}{\sin ^{2} \frac{\pi x_{0}}{L}}\right)\right. & -\frac{\pi^{4} \ell_{1}^{2}}{256 L^{6}}\left[\left(\frac{\phi^{2}}{\pi^{2}}-1\right)^{2}\left(\frac{\phi^{2}}{9 \pi^{2}}-\frac{1}{\sin ^{2} \frac{\pi x_{0}}{L}}\right)-\frac{4 \cos ^{2} \frac{\pi x_{0}}{L}}{\sin ^{6} \frac{\pi x_{0}}{L}}\right] \\
& \left.-\frac{\pi^{2} \ell \ell_{1}}{128 L^{4}}\left[\left(\frac{\phi^{2}}{\pi^{2}}-\frac{1}{3}\right)^{2}-\frac{4}{\sin ^{2} \frac{\pi x_{0}}{L}}\left(\frac{\phi^{2}}{\pi^{2}}+\cot ^{2} \frac{\pi x_{0}}{L}\right)\right]+O\left(\ell^{2} \ell_{1}^{2}\right)\right\} .
\end{aligned}
$$

Here we have neglected the term $\sim \ell^{2} \ell_{1}^{2}$ since it is always much smaller than the term $\sim \ell \ell_{1}$. In Fig. 5 we compare the conductance calculated from Eq. (C17) as a function of $u_{0} a$ with the results of numerical simulations 25].

Exactly at the $p$ resonance, when $\ell_{1} \rightarrow \infty$, correction to the generating function becomes

$$
\delta \mathcal{F}=2 \ln \left[\left(\frac{\phi^{2}}{\pi^{2}}-1\right)^{2}\left(\frac{\phi^{2}}{9 \pi^{2}}-\frac{1}{\sin ^{2} \frac{\pi x}{L}}\right)-\frac{4 \cos ^{2} \frac{\pi x}{L}}{\sin ^{6} \frac{\pi x}{L}}\right] .
$$




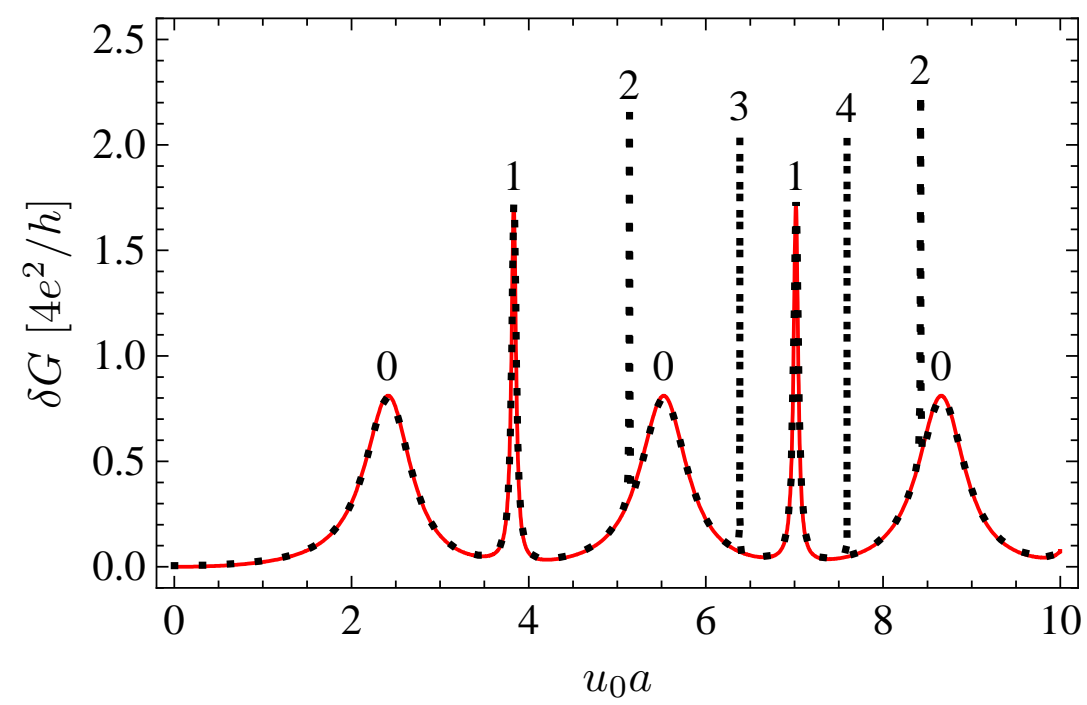

FIG. 5: Correction to the conductance from a circular impurity placed at $x_{0}=L / 2$ as a function of $u_{0} a$. Dotted line: numerical simulations [25] with the parameters $a / L=0.2, W / L=6$; solid line: analytic result including $s$ and $p$ resonances.

From this expression we find the correction to the conductance and average it with respect to the impurity position

$$
\delta G=-\left.\frac{8 e^{2}}{h} \int \frac{d x}{L} \frac{\partial^{2} \delta \mathcal{F}}{\partial \phi^{2}}\right|_{\phi=0}=\frac{32 e^{2}}{9 \pi^{2} h} \int \frac{d x}{L} \frac{\sin ^{4} \frac{\pi x}{L}\left(18+\sin ^{2} \frac{\pi x}{L}\right)}{\left(2-\sin ^{2} \frac{\pi x}{L}\right)^{2}}=\frac{16}{3 \pi^{2}}(15-8 \sqrt{2}) \frac{e^{2}}{h} \approx 1.992 \frac{e^{2}}{h} .
$$

This value is bigger than the conductance correction at the $s$-wave resonance, Eq. (1).

\section{Appendix D: Two-impurity correction}

The second-order correction to the free energy involves the diagrams with two impurities shown in Fig. 6. We sum up these diagrams assuming the two impurities are located at the points $\left\{x, y_{0}\right\}$ and $\left\{x^{\prime}, y_{0}+y\right\}$ and obtain

$$
\delta^{(2)} \mathcal{F}=\frac{1}{2} \ln \operatorname{det}\left\{1-\left[1-T \check{G}_{\text {reg }}(x)\right]^{-1} T \check{G}\left(x, x^{\prime} ; y\right)\left[1-T \check{G}_{\text {reg }}\left(x^{\prime}\right)\right]^{-1} T \check{G}\left(x^{\prime}, x ;-y\right)\right\} .
$$

In the resonant case $T \rightarrow \infty$, this correction to the free energy takes the form

$$
\delta^{(2)} \mathcal{F}=\frac{1}{2} \ln \operatorname{det}\left[1-\check{G}_{\text {reg }}^{-1}(x) \check{G}\left(x, x^{\prime} ; y\right) \check{G}_{\text {reg }}^{-1}\left(x^{\prime}\right) \check{G}\left(x^{\prime}, x ;-y\right)\right] .
$$

With the Green functions calculated above, we evaluate the determinant and obtain the result

$$
\begin{gathered}
\delta^{(2)} \mathcal{F}=\ln \frac{\phi^{4}-\phi^{2}\left(a_{+}^{2}+a_{-}^{2}+b_{+}^{2}+b_{-}^{2}+c_{+}^{2}+c_{-}^{2}\right)+\left(a_{+} a_{-}+b_{+} b_{-}+c_{+} c_{-}\right)^{2}}{\left(\phi^{2}-a_{+}^{2}\right)\left(\phi^{2}-a_{-}^{2}\right)}, \\
a_{ \pm}=\frac{\pi}{\sin \pi(u \pm v)}, \quad b_{ \pm}=\frac{\pi}{\sin \pi(v \pm w)}, \quad c_{ \pm}=\frac{\pi}{\sin \pi(w \pm u)}, \\
u=\frac{x+x^{\prime}}{2 L}, \quad v=\frac{x-x^{\prime}}{2 L}, \quad w=\frac{i y}{2 L} .
\end{gathered}
$$

This expression is to be averaged with respect to the impurity positions. To calculate corrections to the conductance and noise, we expand the free energy in small $\phi$ up to the forth order and integrate each term over $x, x^{\prime}$, and $y$ numerically. Together with the zeroth- and the first-order terms in $n_{\text {imp }}$, this yields

$$
\begin{aligned}
& \overline{\mathcal{F}}=-\frac{W \phi^{2}}{4 \pi L}+ 4 n_{\mathrm{imp}} W L \ln \left(\pi+\sqrt{\pi^{2}-\phi^{2}}\right)+n_{\mathrm{imp}}^{2} W \int_{0}^{L} d x d x^{\prime} \int_{-\infty}^{\infty} d y \delta^{(2)} \mathcal{F} \\
&=\mathrm{const}-\frac{W}{L}\left[\frac{\phi^{2}}{4 \pi}+n_{\mathrm{imp}} L^{2}\left(\frac{\phi^{2}}{\pi^{2}}+\frac{3 \phi^{4}}{8 \pi^{4}}+\ldots\right)-n_{\mathrm{imp}}^{2} L^{4}\left(0.1290 \frac{\phi^{2}}{2}+0.08823 \frac{\phi^{4}}{24}+\ldots\right)\right] .
\end{aligned}
$$




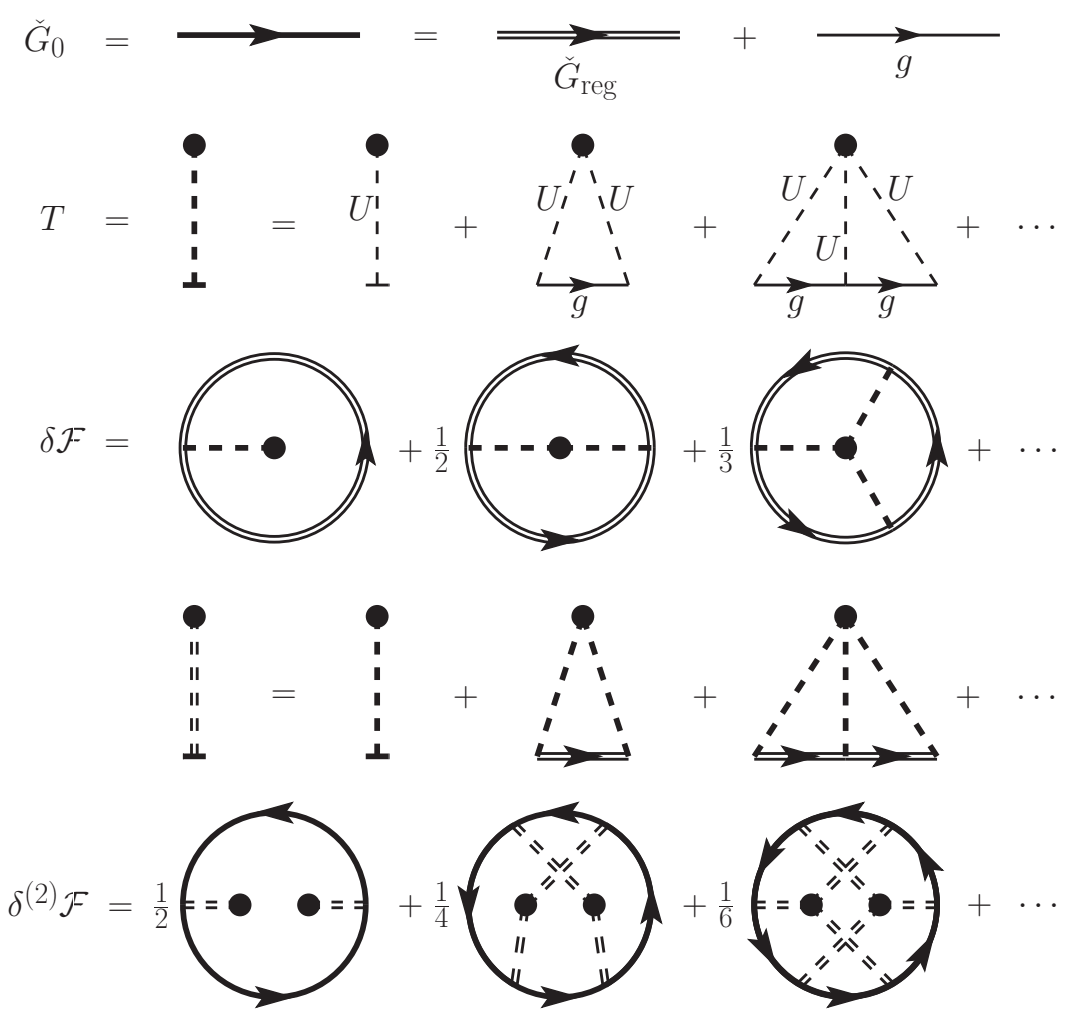

FIG. 6: Feynman diagrams for one- and two-impurity corrections to the free energy.

The conductance and the Fano factor up to the second-order corrections are

$$
\begin{gathered}
G=-\left.\frac{8 e^{2}}{h} \frac{\partial^{2} \overline{\mathcal{F}}}{\partial \phi^{2}}\right|_{\phi=0}=\frac{e^{2} W}{h L}\left[\frac{4}{\pi}+\frac{16}{\pi^{2}} n_{\mathrm{imp}} L^{2}-1.032 n_{\mathrm{imp}}^{2} L^{4}\right], \\
F=\frac{1}{3}-\frac{2}{3} \frac{\partial^{4} \overline{\mathcal{F}} /\left.\partial \phi^{4}\right|_{\phi=0}}{\partial^{2} \overline{\mathcal{F}} /\left.\partial \phi^{2}\right|_{\phi=0}}=\frac{1}{3}-\frac{12}{\pi^{3}} n_{\mathrm{imp}} L^{2}+0.8623 n_{\mathrm{imp}}^{2} L^{4} .
\end{gathered}
$$

